\title{
Preparation and characterization of FA conjuagted MPEG-PLA polymer nanoparticles loaded with 10-hydroxycamptothecin for sustained drug release
}

\author{
Huijuan $\mathrm{Liu}^{1, \mathrm{a}}$, Jinbing Yang ${ }^{3, \mathrm{~b}}$, Xiangrui Yang ${ }^{1, \mathrm{c}}$, Shichao $\mathrm{Wu}^{1,2 \mathrm{~d}}$, Mengmeng \\ $\mathrm{Jia}^{1, \mathrm{e}}$, Liya Xie ${ }^{4, \mathrm{f}}$ and Zhenqing $\mathrm{Hou}^{1, \mathrm{~g}^{*}}$ \\ ${ }^{1}$ Department of biomaterials, College of Materials, Xiamen University, Xiamen 361005, China; \\ ${ }^{2}$ Department of Chemistry, College of Chemistry and Chemical Engineering, Xiamen University, \\ Xiamen 361005, China; \\ ${ }^{3}$ People's Hospital of Xintai City, Xiatai, Shandong 271200, China; \\ ${ }^{4}$ The Firsit Affiliatied Hospital of Xiamen University, Xiamen, 361003, China. \\ a980257495@qq.com, b862076764@qq.com, cyangxiangruix@126.com, 'wushi_happy@126.com,

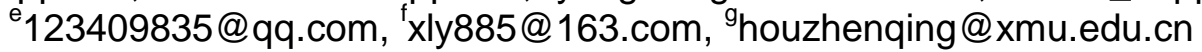 \\ *Corresponding author.: E-mail Address: houzhenqing@xmu.edu.cn
}

Keywords: 10-Hydroxycamptothecin, Self-assembly, PEG-b-PLA, Folate, Nanoparticles.

\begin{abstract}
Suboptimal pharmacokinetic properties, such as poor water solubility, limited stability, and severe side effects, still limited the therapeutic efficacy of drug for cancer chemotherapy, but there has been many strategies to improve these properties. In this paper, we combined several methods in a single drug delivery system and prepared a kind of 10-hydroxycamptothecin (HCPT)-loaded, PEGylated and folate modified polymeric nanoparticles (NPs). The results showed that the HCPT-MPEG-PLA-FA NPs (HMPF NPs) presented a hydrodynamic particle size of about $200 \mathrm{~nm}$, a zeta potential of $-8.9 \mathrm{mV}$, drug encapsulation efficiency of $67.77 \%$, and drug-loading content of 8.23\%. The in vitro drug release experiments exhibited a slow and prolonged release profile over 160 h. These results suggested that the HMPF NPs were well suited for drug delivery.
\end{abstract}

\section{Introduction}

Nowadays, cancer has become one of the most common causes of death around the world, and chemotherapy is still a commonly used strategy in cancer therapy. ${ }^{[1]}$ However, most anticancer drugs show poor water solubility, limited stability, rapid blood clearance and severe side effects, which largely limit their clinic application. ${ }^{[2]}$ In recent years, there are numerous strategies for optimizing these suboptimal pharmacokinetic properties. ${ }^{[3]}$ Engineering the poorly soluble drugs with carrier materials and surfactants into drug nanoparticles (NPs) provides numerous routes to improve its bioavailability. ${ }^{[4,5]}$ With the protection of the carrier and the surfactant, NPs can not only increase the stability of the drug, but also slowly release the drug and prolong half-life of the drug. It is well known that the PEGylation was favored for water dispersibility and stability. ${ }^{[6]}$ Hence, it is a very great progress to fabricate the drug into NPs.

Among all the suboptimal pharmacokinetic properties, severe side effects were the biggest problem in clinic application. ${ }^{[7]}$ It was because the anticancer drug would attack the healthy cells as well as the cancer cells. Hence, improving the drug targeting property would decrease the side effect. It has long been believed that folate (FA) is a good target molecule because of the over-expression FA receptors in cancer cells. ${ }^{[8,9]}$ The NPs with the functionalization of FA could latch onto FA receptors of the cancer and thus enhance their cellular uptake. Herein, we used a dialysis technique to prepared the HCPT loaded, MPEG-PLA-FA (monomethoxy polyethylene glycol-polylactide-folate) modified polymeric NPs, which possessed improved the drug sustained release properties. The characteristics of MPEG-PLA-FA copolymer were evaluated by Fourier transform infrared spectroscopy (FT-IR). 
The physicochemical characteristics of the self-assembled HMPF NPs including particle size, zeta potential, morphology and drug release were characterized by dynamic light scattering (DLS), electrophoretic light scattering (ELS), scanning electron microscopy (SEM), transmission electron microscopy (TEM) and fluorescence spectrophotometry.

\section{Methods}

\subsection{Materials.}

All chemicals were analytical grade and used as received without further purification. The ultrapure water $\left(18 \mathrm{M} \Omega \cdot \mathrm{cm}^{-1}\right)$ was used throughout the work. The 10-HCPT (purity $>99 \%$ ) was purchased from Lishizhen Pharmaceutical Co., Ltd. The monomethoxy (polyethylene glycol)-poly (lactide-co-glycolide) (MPEG-PLA, PEG: 10\%, 5000 Da, PLA: 28000 Da, 85/15) was obtained from Daigang Biotechnology Co., Ltd.

\subsection{Preparation of the MPEG-PLA-FA conjugation.}

MPEG-PLA-FA was synthesized via the esterification reaction between the carboxyl group of FA molecule and the hydroxyl group (derived from PLA) of MPEG-PLA polymer. 0.025 mmol of FA dissolved in $10 \mathrm{~mL}$ of dimethylsulfoxide (DMSO) was reacted with $0.05 \mathrm{mmol}$ of NHS and 0.05 mmol of DCC under nitrogen atmosphere at room temperature for $12 \mathrm{~h}$. The activated folic acid was reacted with $0.012 \mathrm{mmol}$ of MPEG-PLA dissolved in $5 \mathrm{~mL}$ of DMSO. The reaction was performed under nitrogen atmosphere at room temperature for $4 \mathrm{~h}$. The mixture was filtered and then dialyzed against PBS/DI water to remove excess FA and other byproducts and lyophilized for 24 .

\subsection{Preparation of the HMPF NPs.}

The HMPF NPs were prepared by a facile dialysis method. In brief, $100 \mathrm{mg}$ of MPEG-PLA-FA and $10 \mathrm{mg}$ of HCPT were dissolved in $10 \mathrm{~mL}$ of DMSO, and the mixture was used as the organic phase. Subsequently, the resulting organic phase was then introduced into a dialysis bag and dialyzed against 1, $000 \mathrm{~mL}$ of water as the aqueous phase for $8 \mathrm{~h}$. The HMPF NPs were lyophilized for $24 \mathrm{~h}$ using a freeze drier and stored at $4^{\circ} \mathrm{C}$ for use. The HMP NPs were prepared in a similar way by using MPEG-PLA instead of MPEG-PLA-FA.

\subsection{Characterization.}

The morphology of HMPF NPs was examined by SEM (UV-70) at $10 \mathrm{kV}$ and by TEM (JEM-2100) at $200 \mathrm{kV}$. The average particle size and size distribution of the NDs were determined by photon correlation spectroscopy with a Malvern Zetasizer Nano-ZS (Malvern Instruments, Malvern) at $25^{\circ} \mathrm{C}$ under suitable dilution conditions. Measurements were repeated for three times to get the consistent results. The amount of HCPT entrapped in the particles was determined indirectly by fluorescence spectrophotometry (Fluoromax-4). All samples were assayed at $383 \mathrm{~nm}$. The weight of the drug entrapped in the particles was calculated by the calibration curve. ${ }^{[4]}$

\subsection{In vitro drug release studies.}

The in vitro drug release studies of particles were performed using the dialysis technique. The HCPT-loaded NPs were dispersed in PBS $(10 \mathrm{~mL})$ and placed into a pre-swelled dialysis bag (MWCO $3500 \mathrm{Da}$ ). The dialysis bag was then immersed in 0.1 M PBS at pH 6.8, 7.4, and 8.0, and oscillated continuously in a shaker incubator $(100 \mathrm{rpm})$ at $37^{\circ} \mathrm{C}$. All samples were assayed by fluorescence spectrophotometry. Free HCPT at the equivalent concentrations were used for comparison.

\section{Results and discussion}

\subsection{Preparation and characterization of the HMPF NPs.}

First, we synthesized the MPEG-PLA-FA conjugation via the esterification reaction between the carboxyl group of FA molecule and the hydroxyl group (derived from PLA) of MPEG-PLA polymer (Fig. 1A). Fig. 1B shows the FT-IR spectra of MPEG-PLA and MPEG-PLA-FA. Compared to MPEG-PLA, signal intensity at $1744 \mathrm{~cm}^{-1}$ clearly increased in the IR spectrum of MPEG-PLA-FA, corresponding to $\mathrm{C}=\mathrm{O}$ stretching vibration of ester bond. In addition, the characteristic peaks of $\mathrm{FA}$ at 
$2561 \mathrm{~cm}^{-1}$ and $666 \mathrm{~cm}^{-1}$ appeared in the IR spectrum of MPEG-PLA-FA, corresponding to hydroxy and benzene rings of FA. These results indicated FA was successfully conjugated to hydroxyl group of MPEG-PLA via ester bond.
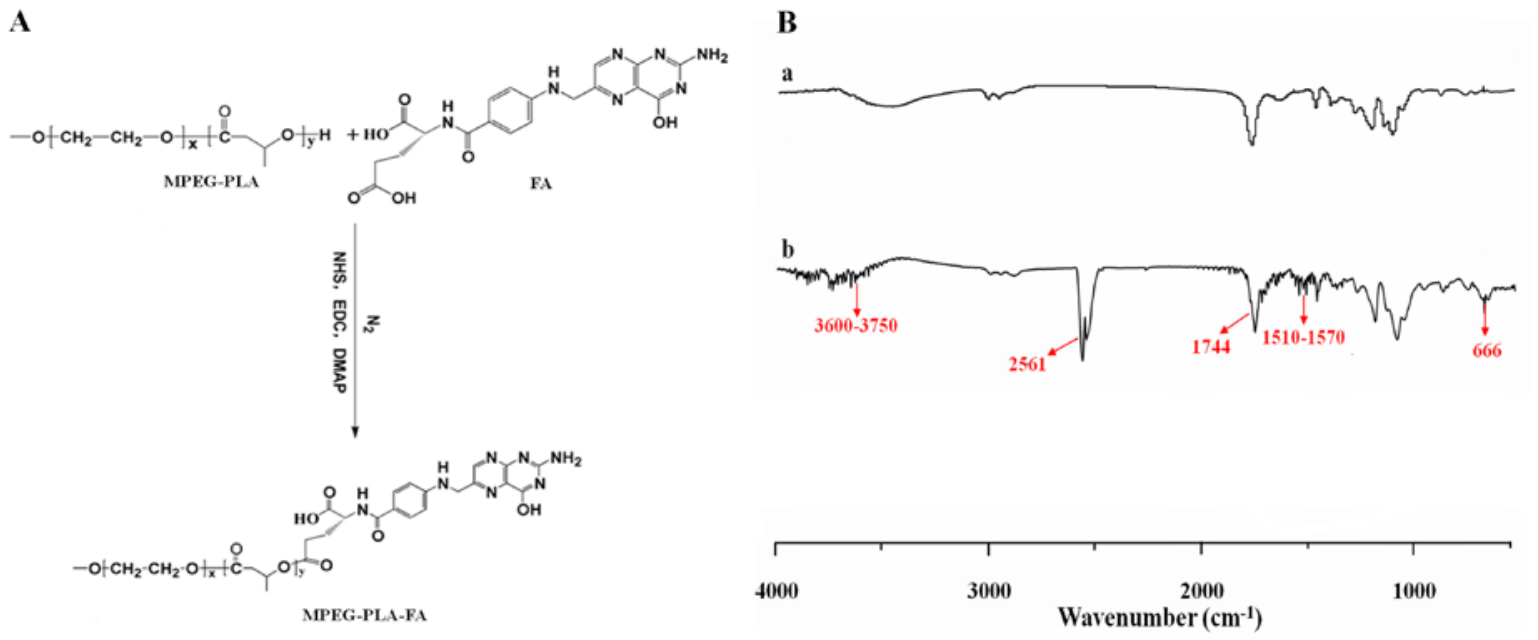

Fig. 1. Synthesis pathway of MPEG-PLA-FA (A) and the FT-IR spectra (B) of MPEG-PLA (a) and MPEG-PLA-FA (b).

The HMPF NPs was prepared via the dialysis method. Both HCPT and MPEG-PLA-FA were dissolved in dimethyl formamide (DMF), and the mixture was dialyzed against water for $6 \mathrm{~h}$. Due to the good miscibility between DMF and water, DMF was gradually removed and slowly replaced with water in the dialysis process. To attain the minimal energy state, the hydrophobic PLA and HCPT assembled together and formed a core, while the hydrophilic PEG and FA extended to the aqueous environment to form a shell.

The morphology of HMPF NPs were evaluated by SEM and TEM. The SEM image (Fig. 2A) shows that the HMPF NPs are spherical with a relatively smooth surface. The TEM image (Fig. 2B) and the result of DLS shows that the NPs are homogeneous solid particles with an average diameter of about $230.4 \pm 8.7 \mathrm{~nm}$ and a zeta potential of $-8.9 \pm 0.8 \mathrm{mv}$. The determined drug entrapment efficiency and drug-loaded content of HMPF NPs determined by fluorescence spectrophotometry were $67.77 \pm$ $0.57 \%$ and $8.23 \pm 0.07 \%$, respectively.

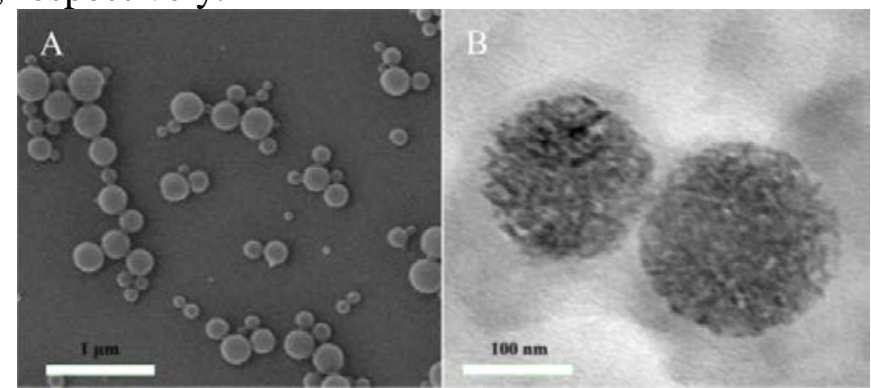

\subsection{In vitro drug release behavior.}

Fig. 2. SEM image (A) and TEM image (B) of HMPF NPs

The in vitro release study of the HMPF NPs was performed in PBS at $\mathrm{pH} \mathrm{6.8,} \mathrm{7.4,} \mathrm{and} \mathrm{8.0.} \mathrm{As}$ shown in Fig. 3, the HMPF NPs exhibited a slow, prolonged release profile over a period of $160 \mathrm{~h}$ and there was no dramatic initial burst release. This was because the HCPT was encapsulated in the inner part of the nanoparticles. The release of HCPT molecules was maximum and the fastest at $\mathrm{pH}$ 8.0. Because HCPT would open the ring structure in the alkaline $\mathrm{pH}$ environment to carboxylate form and the solubility would greatly increase. But it still exhibited a controlled release manner, which demonstrated that the HMPF NPs could protect HCPT and thus prolong its half-life. 


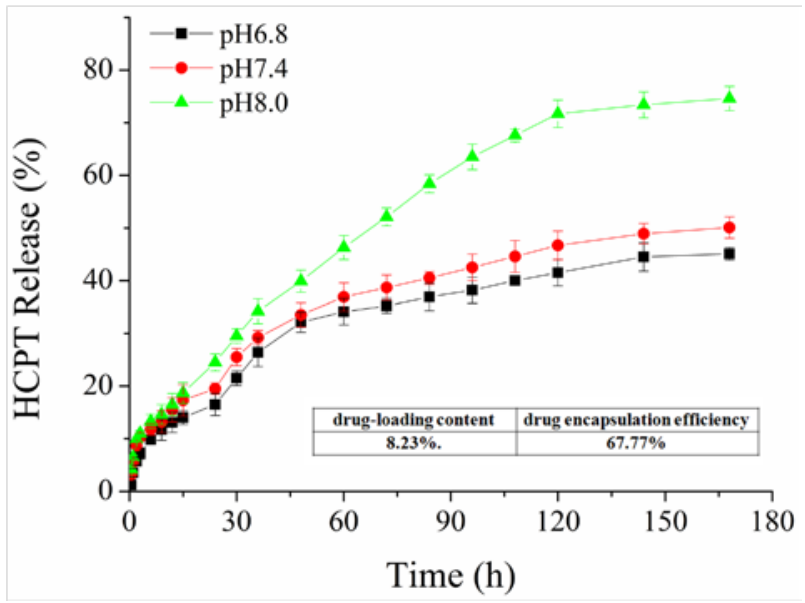

Fig. 3. In vitro release profiles of HMPF NPs at $\mathrm{pH}$ 6.8, 7.4, and 8.0.

\section{Conclusions}

In this study, we developed a simple but successful method to obtain the HMPF NPs with fine characteristics for drug delivery. The HMPF NPs presented better dispersibility and stability properties and drug sustained release properties. The results obtained in this study indicate that these NPs might become a promising drug delivery system for HCPT.

\section{Acknowledgements}

The project was supported by the Natural Science Foundation of Fujian Province of China

(No.2016J01406) and Fujian Province medical innovation project 2014-CXB-350

\section{References}

[1] Riehemann K, Schneider SW, Luger TA, et al. Nanomedicine-Challenge and Perspectives. Angew. Chem. Int. Ed. Vol. 48 (2009), p. 872-897.

[2] Kolate A, Baradia D, Patil S, et al. PEG-A versatile conjugating ligand for drugs and drug delivery systems. J. Controlled Release. Vol. 192 (2014), p. 67-81.

[3] Kamaly N, Xiao ZY, Valencia PM, et al. Targeted polymeric therapeutic nanoparticles: design, development and clinical translation. Chem. Soc. Rev. Vol. 41 (2012), p. 2971-3010.

[4] Jiang W, Kim BYS, Rutka JT, et al. Nanoparticle-mediated cellular response is size-dependent. Nat. Nanotechnol. Vol. 3 (2008), p. 145-150.

[5] Sahay G, Alakhova DY, Kabanov AV. Endocytosis of nanomedicines. J. Controlled Release. Vol. 145 (2010), p. 182-195.

[6] Harris JM, Chess RB. Effect of pegylation on pharmaceuticals. Nat. Rev. Drug Discovery. Vol. 2 (2003), p. 214-221.

[7] Prabaharan M, Grailer JJ, Pilla S, et al. Gold nanoparticles with a monolayer of doxorubicin-conjugated amphiphilic block copolymer for tumor-targeted drug delivery. Biomaterials. Vol. 30 (2009), p. 6065-6075.

[8] Chan P, Kurisawa M, Chung JE, et al. Synthesis and characterization of chitosan-g-poly(ethylene glycol)-folate as a non-viral carrier for tumor-targeted gene delivery. Biomaterials. Vol. 28 (2007), p. 540-549.

[9] Mansouri S, Cuie Y, Winnik F, et al. Characterization of folate-chitosan-DNA nanoparticles for gene therapy. Biomaterials. Vol. 27 (2006), p. 2060-2065. 